\title{
Article
}

\section{Stakeholders' views on granting prescribing authority to pharmacists in Nigeria: a qualitative study}

\author{
Auta, Asa, Strickland-Hodge, Barry and Maz, Julia \\ Available at http://clok.uclan.ac.uk/14626/ \\ Auta, Asa ORCID: 0000-0001-6515-5802, Strickland-Hodge, Barry and Maz, \\ Julia (2016) Stakeholders' views on granting prescribing authority to \\ pharmacists in Nigeria: a qualitative study. International Journal of Clinical \\ Pharmacy, 38 (4). pp. 960-967. ISSN 2210-7703
}

It is advisable to refer to the publisher's version if you intend to cite from the work. http://dx.doi.org/10.1007/s11096-016-0321-6

For more information about UCLan's research in this area go to http://www.uclan.ac.uk/researchgroups/ and search for <name of research Group>.

For information about Research generally at UCLan please go to http://www.uclan.ac.uk/research/

All outputs in CLoK are protected by Intellectual Property Rights law, including Copyright law. Copyright, IPR and Moral Rights for the works on this site are retained by the individual authors and/or other copyright owners. Terms and conditions for use of this material are defined in the policies page.

\section{CLoK}

Central Lancashire online Knowledge www.clok.uclan.ac.uk

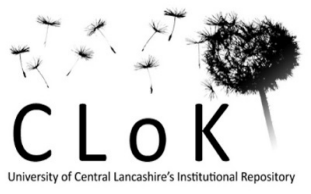


Stakeholders' views on granting prescribing authority to pharmacists in Nigeria: a qualitative study

Asa Auta ${ }^{1}$,

${ }^{1}$ School of Pharmacy and Biomedical Sciences, University of Central Lancashire, Preston, PR1 2HE, UK

Barry Strickland-Hodge $\mathrm{e}^{2,3}$,

${ }^{2}$ School of Healthcare, Faculty of Medicine and Health, University of Leeds, Leeds, LS2 9JT, UK.

${ }^{3}$ Centre for Technology Enabled Health Research, Faculty of Health and Life Sciences, Coventry University, Coventry, CV1 5FB, UK

Julia Maz',

${ }^{2}$ School of Healthcare, Faculty of Medicine and Health, University of Leeds, Leeds, LS2 9JT, UK.

Corresponding author: Asa Auta, School of Pharmacy and Biomedical Sciences, University of Central Lancashire, Preston, PR1 2HE, UK. Email: aauta@uclan.ac.uk 


\begin{abstract}
Background: In Nigeria, only medical doctors, dentists and some nurses in primary care facilities have the legal right to prescribe medicines to patients. Patients' access to prescription medicines can be seriously affected by the shortage of prescribers leading to longer waiting times in hospitals.
\end{abstract}

Objective: This research was carried out to investigate stakeholders' views on granting prescribing authority to pharmacists in Nigeria.

Setting: The study was conducted in Nigeria

Methods: Qualitative, semi-structured interviews were conducted with 43 Nigerian stakeholders including policymakers, pharmacists, doctors and patient group representatives. Transcribed interviews were entered into the QSR NVivo 10 software and analysed using a thematic approach.

Main outcome measure: Stakeholders' perception on the granting of prescribing authority to pharmacists in Nigeria.

Results: Three major themes emerged from the interviews: 1) prescribing as a logical role for pharmacists, 2) pharmacist prescribing- an opportunity or a threat and 3) the potential barriers to pharmacist prescribing. Many non-medical stakeholders including pharmacists and patient group representatives supported an extended role for pharmacists in prescribing while the majority of medical doctors including those in policy making were reluctant to do so. Generally, all stakeholders perceived that pharmacist prescribing represents an opportunity to increase patients' access to medicines, reduce doctors' workload and promote the utilisation of pharmacists' skills. However, many stakeholders including pharmacists and doctors commonly identified pharmacists' inadequate skills in diagnosis, medical resistance and shortage of pharmacists as potential barriers to the introduction of pharmacist prescribing in Nigeria.

Conclusion: The present study showed a split of opinion between participants who were medical doctors and those who were non-doctors in their support for pharmacist prescribing. However, all stakeholders acknowledged the potential of pharmacist prescribing to increase patients' access to medicines in Nigeria.

Keywords: pharmacist prescribing; access to medicines; physicians; pharmacists; policymakers; patient representatives; Nigeria 


\section{Impact of findings on practice}

- Patients' access to prescription medicines can be seriously affected by the shortage of prescribers in Nigeria.

- Pharmacists in Nigeria are highly educated professionals whose skills have been underutilised.

- Pharmacist prescribing offers an opportunity to increase patients' access to medicines in Nigeria and promote the utilisation of pharmacist skills.

\section{Introduction}

To prescribe is to "authorize by means of a written prescription the supply of a medicine" [1 p.11]. Prescribing to patients was the exclusive role of doctors and dentists, until the introduction of non-medical prescribing (NMP) in some countries which allowed non-medical professionals including nurses and pharmacists to prescribe medicines [2, 3]. A number of countries have legislated for NMP including the United States of America (USA), United Kingdom (UK), Australia, New Zealand, Canada, Sweden, Republic of Ireland, South Africa, Botswana, Kenya and Uganda [4]. Nurse prescribing is the most popular form of NMP. Available data shows that Nurses have been authorised to prescribe in 22 countries [4]. Pharmacist prescribing is increasingly being accepted and implemented in many developed countries. Pharmacists currently have the legal right to prescribe medicines in the USA, UK, Canada, New Zealand and Israel [5-7]. The increase in the international recognition of pharmacist prescribing has been facilitated by the desire to ensure prompt patients' access to prescription medicines and better utilisation of pharmacists' skills [8].

Nevertheless, in many developing countries including Nigeria, where access to medicines has remained a public health challenge due to shortage of medical prescribers, pharmacist prescribing is yet to be recognised as an important strategy that would enhance patients' access to medicines. The legislation concerning prescribing of medicines in Nigeria states that:

"In tertiary and secondary health care institutions, only duly qualified and licensed medical practitioners shall have the authority to prescribe drugs. At the primary health care level, government shall designate appropriate health care personnel to prescribe drugs; only qualified and licensed medical practitioners shall have the authority to prescribe drugs in the private sector" [9 p.25].

Hence, only medical doctors and dentists have the legal right to prescribe medicines to patients in Nigeria, except in primary health centres (PHCs). Since most PHCs lack doctors, prescribing is mostly done by nurses and community health workers using standing orders or 
protocols $[10,11]$. However, a number of concerns exist in terms of the safety of prescribing by nurses and community health workers because of their lack of adherence to treatment guidelines and inadequate knowledge of medicines $[10,12,13]$.

Therefore, the general out-patient department (GOPD) of hospitals in Nigeria is usually the entry points for most patients seeking medical help because of the lack of doctors in PHCs $[14,15]$. Because of this lack of doctors, a doctor in a GOPD in Nigeria has approximately 40 - 50 patient consultations per day [16, 17]. There are about 40 doctors per 100,000 people in Nigeria compared with 280 doctors per 100,000 people in the UK including general practitioners and specialist physicians [18]. Furthermore, more than $50 \%$ of patients visiting GOPDs wait for between one to three hours to see a doctor $[16,17,19]$. Thus, it is beyond doubt that the current prescribing arrangements in Nigeria do not sufficiently meet the needs of patients in terms of timely and convenient access to prescriptions.

Pharmacists in Nigeria are highly educated professionals with expertise in medicines management. Their mode of training follows similar models in advanced countries including the UK [20]. It consists of a five-year undergraduate professional programme followed by a one-year pre-registration work-based supervised training [20]. In addition, many pharmacists in Nigeria are specialising as clinical pharmacists at various universities and the West African Postgraduate College of Pharmacists [21]. Despite the cost and high level of training of pharmacists in Nigeria, their expertise is not used effectively as their roles have not been expanded to a more clinical one. They mainly perform the traditional role of dispensing, which could be handled by suitably trained pharmacy technicians as in other countries such as the UK [22].

Pharmacists in Nigeria have shown a willingness for expanded clinical roles including prescribing [23, 24]. In addition, a pilot survey conducted among clients of community pharmacists in a city in Nigeria has revealed a high support for pharmacist prescribing [25]. However, no research has explored the views of other key stakeholders including policymakers, leaders of professional medical and pharmacy groups, who are the major players in driving medicines policies in Nigeria. Therefore, this necessitated this research to explore the opinions of various stakeholders involved in medicines prescribing on the potential for pharmacist prescribing in Nigeria.

\section{Aim of the study}

This study was carried out to explore the views of stakeholders involved in prescribing and pharmacy practice on the extension of pharmacists' clinical roles to include prescribing in Nigeria. 


\section{Ethics approval}

This study required and received ethics approval from the School of Healthcare Research Ethics Committee, University of Leeds, UK and the Plateau State Specialist Hospital Health Research Ethics Committee, Nigeria. All participants were provided with an information sheet containing details of the research and written or oral, informed consent was obtained from them.

\section{Methods}

Using a purposeful sampling technique employing maximum variation in order to obtain a range of perspectives (mix of stakeholders, practice and geographical settings), generic recruitment emails were sent to 134 prospective participants. These included heads of pharmacy and medical departments in some secondary and tertiary hospitals; leaders of professional pharmacy and medical associations including the Pharmaceutical Society of Nigeria and Nigerian Medical Association; directors of pharmaceutical and medical departments at the State and Federal Ministry of Health; academics and patient group representatives. A total of 43 stakeholders who accepted to participate in the study were interviewed.

Qualitative semi-structured telephone interviews were conducted from April to June 2014. Telephone interviews were employed to enable the interviewing of a range of stakeholders across many locations in Nigeria. The interview guide was based on a study conducted in England and the review of the literature [22]. Pharmacist prescribing from the perspective of this research was introduced to participants to mean the granting of pharmacists the legal authority to prescribe 'Prescription Only Medicines' as currently practised by doctors in Nigeria which differs from counter prescribing or the supply of over the counter medicines as currently practised by community pharmacists in response to patients' complaints or symptoms. Topic areas covered included participants' perception of pharmacist prescribing, reservations concerning pharmacist prescribing, potential facilitators and barriers to pharmacist prescribing in Nigeria, advantages associated with pharmacist prescribing and the potential changes in the structure of pharmacy that would be needed for the development of pharmacist prescribing in Nigeria. The interviews conducted lasted between 20 to 70 minutes. Data saturation was achieved when further interviews did not yield any new thematic information and this was perceived to be reached at about the 35th interview. However, further interviews were carried out with the remaining eight stakeholders who accepted to participate in the study.

The interviews conducted were digitally recorded and fully transcribed. One researcher checked each transcript against the digital audio recording for accuracy. Transcript data were 
entered into the QSR NVivo 10 software for appropriate data management. Data were analysed using thematic analysis in the manner outlined by Braun and Clarke [26]. The analysis proceeded in iterative stages of data familiarisation, generation of codes, searching, reviewing, defining and naming of themes. The data were independently coded and analysed by one researcher but reviewed at different stages by two additional researchers including reviewing and refining the coding structure and identified themes. At the final stage of the analysis, identified themes were discussed by the three researchers until a consensus was reached.

\section{Results}

\section{Demographics}

The 43 stakeholders interviewed comprised of 20 pharmacists, 9 doctors, 11 policymakers, and 3 patient group representatives. These participants were drawn from the six geopolitical regions of Nigeria. The 11 policymakers interviewed were from the federal (5) and state (6) levels. The 20 pharmacists who participated in this study included 4 academics, 5 community pharmacists and 11 hospital pharmacists; while the 9 doctors were 4 consultants, 3 residents, and 2 general practitioners. The pharmacists interviewed had from 5 to 37 years of experience while the doctors had from 10 to 37 years of experience. Eight (8) of the participants interviewed were practising in secondary hospitals located in rural centres.

\section{Views on pharmacist prescribing}

Three themes emerged from stakeholders' views on pharmacist prescribing in Nigeria:

1. Prescribing as a logical role for pharmacists

2. Pharmacist prescribing- an opportunity or a threat

3. The potential barriers to pharmacist prescribing

These themes are reported below.

\section{Prescribing a logical role for pharmacists}

Generally, opinions were split between pharmacists and doctors concerning pharmacist prescribing. Pharmacists were generally in support of a prescribing authority for them. They saw it as a logical role for pharmacists given their knowledge of medicines.

"But we know when we go to the hospitals, let say a general hospital in the rural area, the prescribers really are not doctors, they are either senior nursing or community health officers. So why not allow the pharmacists to also prescribe, more so that he knows the drugs and now with his training [clinical] 
he knows some of the diseases with their clinical features." P26, Academic Pharmacist

Some community pharmacists reported that they do counter prescribing in response to patients' complaints and therefore saw prescribing as a natural progression of their roles.

"I attend to patients. They come with minor ailments and I treat them. So, I don't see prescribing as a difficult thing to do." P08, Community Pharmacist

Similarly, a hospital pharmacist interviewed also considered prescribing as a natural progression of hospital pharmacists' roles. This pharmacist reported that they work collaboratively with doctors to influence doctors' prescribing.

"Sometimes we suggest to the doctor what to prescribe. So, what is stopping us from prescribing?" P18, Hospital Pharmacist

However, many doctors were non-supportive of a prescribing role for pharmacists.

"On a personal note I think pharmacists should not prescribe. I think it is not part of their job, they shouldn't prescribe." P29, Resident Doctor

Interviews held with policymakers also reiterated the split of views observed between doctors and pharmacists as policymakers views were aligned to their professional background.

"In my own opinion, I think they [pharmacists] are well equipped to be granted the right to prescribe. $P 27$, Policymaker with a pharmacy background

“No, no, no, no, I don't support that. Any person that should be allowed to give a prescription should be a medical doctor but for a pharmacist to be a prescriber, I don't support that." P36, Policymaker with a medical background

Despite many doctors' lack of support to pharmacist prescribing, a few of them reported that granting some form of prescribing authority to pharmacists will be a logical thing to do.

"You know, hospital facilities are not everywhere. Given our health challenges, there is need to empower pharmacists to be able to do more than they are doing at the moment." P17, General Practitioner

Furthermore, patient group representatives interviewed also felt that prescribing would be a reasonable role for pharmacists and were supportive of pharmacist prescribing. 
"Why not? Even nurses who do not have the amount of knowledge that pharmacists have on drugs, prescribe. So why can't the pharmacist prescribe?" P38, Patient group representative

\section{Potential barriers to pharmacist prescribing}

Participants identified a number of factors that could hinder the introduction of pharmacist prescribing in Nigeria. Many participants including doctors, pharmacists, policymakers and patient group representatives expressed concern over pharmacists' skills in diagnosis. They saw that as a potential barrier.

"Yeah, the concern about pharmacist prescribing will arise basically from the fact that the competence to be able to make accurate diagnosis is critical in therapy." P17, Medical doctor

However, if pharmacists are to prescribe in collaboration with doctors as suggested by many participants, the concern of pharmacists' inadequate skills in diagnosis would be taken care of.

"I think in Nigeria the best way for now is to do it in collaboration with a doctor because they are trained to diagnose." P11, Hospital Pharmacist

Nevertheless, participants observed that collaborative prescribing will only be practicable in hospital settings where doctors and pharmacists work together in patient care. They reported that this is unlikely in community pharmacy settings given that there is currently no structure in place that allows community pharmacists to work in partnership with doctors.

Generally, resistance from the medical profession was cited as a fundamental barrier to pharmacist prescribing in Nigeria. Participants felt that such resistance is expected because doctors would react in such a way to protect their territory.

"If you tell Nigerian doctors to relinquish what they see as their birth right to another professional, there will be serious opposition" P03, Pharmacist

Many pharmacists felt that medical resistance is likely to occur at policy level because of the overriding dominance of doctors in the leadership structure of health sector.

"If you look at the federal and state ministry of health, the top hierarchies are occupied by doctors. So, policy wise there is also a barrier." P32, Policymaker Another major barrier mentioned was the shortage of pharmacists in many hospitals. 
"So, hospital pharmacists are overwhelmed by the traditional pharmacy responsibilities of dispensing medications and procuring drugs. So, they may not have the time to do an additional role but if they are more in number that will be facilitated." P26, Pharmacist

There were concerns that pharmacist's attitude in taking up extended clinical roles could be a possible barrier to pharmacist prescribing as mentioned by a participant below.

“Pharmacists' attitude is usually a big barrier. Some pharmacists are very comfortable with those traditional roles, they don't want to acquire new skills, and they don't want to move into different areas where pharmacists are needed in clinical practice and all that." P05, Pharmacists

However, the popular view held by many pharmacists indicated that many of them are unsatisfied with their current roles and were willing to expand their roles clinically. Such willingness for role expansion could be a driver to extended role in prescribing.

"Yes, the younger pharmacists are willing to expand their roles clinically, even some older ones too. The younger pharmacists because of their mode of training they see themselves as being under-utilised." P16, Pharmacist

Furthermore, some participants expressed concern that community pharmacists may have conflict of interest in their prescribing since many community pharmacies were established on a business model.

"If the pharmacist is allowed to prescribe in his community pharmacy, there will be a lot of problems because the pharmacist will only be interested in the patient buying his drugs and this can bias his prescription." P06, Pharmacist

Also, some participants mentioned that community pharmacists may not have access to patients' medical records and adequate facilities that would support their prescribing practice.

\section{Pharmacist prescribing: an opportunity or a threat}

While many participants saw the granting of prescribing authority to pharmacists as beneficial; others perceived it as a threat.

Generally, there was a perception among many stakeholders that allowing pharmacists to prescribe could improve patients' access to treatment and reduce waiting time.

"You see, in some facilities there are no doctors at all. Allowing pharmacists

to prescribe will go a long way." $P 24$, Patient group representative 
"The patient time will be saved because the patient will not have to wait all day waiting for a doctor's prescription" P2, General Practitioner

A participant also noted that it will increase the options in which patients have to seek treatment from different professionals, thereby empowering the patient.

"You will have more experts prescribing to patients rather than just the doctor." P27, Policymaker

Participants, particularly pharmacists observed that pharmacist prescribing will promote the utilisation of pharmacists' skills, make pharmacists job more satisfying and enhanced pharmacists' professional status.

"Definitely, there will be job satisfaction on the part of the pharmacists. I am very, very satisfied doing clinical work." P05, Hospital Pharmacist

A medical doctor also expressed similar view on the potential benefit to the pharmacy profession.

"I think it will be a lot of fun for them. Pharmacists will be able to have patients that they treat from beginning to the end of the disease process all on their own and that will be quite an exciting experience and might inspire them to be even more professional about their practice and of course the satisfaction they will derive from all of that I think all will add up to improving perhaps the practise so to say of the pharmacist in the public eye." P17, General Practitioner

There was a general view among all stakeholders that pharmacist prescribing in Nigeria could reduce doctors' workload and free them up to concentrate on other specialised tasks

"It will take the pressure off the general practitioner which means that the doctors will now concentrate on more delicate issues of diagnosis instead of having to be battling with minor illness." P31, Policymaker

However, some participants particularly doctors viewed pharmacist prescribing as an encroachment into doctors' professional boundary as prescribing has always been the doctors' role in Nigeria. Therefore, these doctors responded in such a way to preserve this boundary.

"No, I will not support pharmacists prescribing. They should do their own work. Prescription is under the boundary of the medical doctors. I mean it is one of the main things we have as doctors, by the time every other person prescribes, 
then what does the doctor really do? So, that will be like taking our jobs from us." P33, Policymaker and Medical Doctor

Furthermore, a few pharmacists were concerned that pharmacist prescribing might result in the loss of the exclusive right they have over dispensing. They fear that doctors are likely to seek for some dispensing authority if pharmacists are allowed to prescribe.

“... if we pursue that objective, the doctors too may want to say let us too have some dispensing role which is traditionally the function of the pharmacists." P03, Hospital Pharmacist

This concern was expressed by pharmacists who viewed dispensing as a key role that maintains pharmacists' professional status.

\section{Discussion}

This study showed a split of opinion between participants who were medical doctors and those who were non-doctors. While many non-medical stakeholders interviewed including pharmacists and patient group representatives supported an extended role for pharmacists in prescribing, many medical doctors including those in policy making were reluctant to do so.

Many pharmacists interviewed, including those who are policymakers considered prescribing as a logical role for pharmacists considering pharmacists' knowledge in therapeutics. This knowledge they considered essential for prescribing. However, prescribing is a complex process that encompasses identifying patients' problem; specifying treatment objectives; determining the safe and cost-effective treatment; instituting and monitoring patients' therapy [27]. These processes involve the use of the prescribers' knowledge and skills in diagnosis and therapeutics to make informed clinical decisions concerning patients' therapy [28]. Unlike the medical practitioners' training, pharmacists' basic training is limited in clinical assessment and diagnosis. In many countries where pharmacists are legally allowed to prescribe including the UK and New Zealand additional training is provided to prepare pharmacists for prescribing $[3,28]$. The training incorporates applied therapeutics and differential diagnosis; and a period of learning in practice under a medical mentor [3]. In addition, these countries implemented a collaborative form of prescribing initially which does not require the pharmacist to make diagnostic decisions. Therefore, a collaborative form of prescribing, as suggested by participants of this study would be good for initial implementation in Nigeria. This form of prescribing would appeal to doctors as the diagnosis, overall patient management and initial treatment decisions remain with the medical prescriber [28]. Also, certain benefits are likely to be associated with a collaborative prescribing in Nigeria. It will foster collaboration between 
doctors and pharmacists and provide the prescribing pharmacist the opportunity to learn from the medical prescriber especially in the aspect of diagnosis. The experience gained could be instrumental if independent prescribing is subsequently implemented.

Generally, many medical doctors were unsupportive for pharmacist prescribing because prescribing in Nigeria is under their jurisdiction. Therefore they viewed pharmacist prescribing as an encroachment into medical territory and a challenge to medical dominance. This finding is unsurprising because similar attitudes were shown by medical doctors in other countries including South Africa and Canada where pharmacists have attempted to expand their roles to include prescribing $[29,30]$. Doctors in these countries responded in such a way to protect their professional territory from encroachment $[29,30]$. The general behaviour of medical doctors could be explained by the sociological theory that posit that prescribing is a core role that confers clinical autonomy to medical doctors and also grants them control over the role of other healthcare professionals [31, 32]. For example, a pharmacist's role of dispensing is dependent on an instruction (prescription) from a doctor. This therefore puts the medical doctor in control of the work sphere of the pharmacist [32]. Hence, the fear of the loss of this control could account for doctors' non-support for pharmacist prescribing.

A major factor that gained support for pharmacist prescribing among participants was the potential benefits associated with it. Patient group representatives were very supportive of a prescribing authority for pharmacist because they perceived it will promote quick and convenient access to treatment. Other stakeholders including medical doctors and policymakers also acknowledged that pharmacist prescribing could potentially increase patients' access to treatment in Nigeria. Increasing patients' access to care while maintaining patient safety has primarily been the driver for NMP including pharmacist prescribing in many countries where it has been introduced $[1,33]$. Many research reports from the UK have indicated that patients' access to treatment has been enhanced as a result of non-medical prescribing $[34,35]$. This would be of great benefit to patients in Nigeria. For example, about 110 million malaria cases are clinically diagnosed annually in Nigeria and uncomplicated malaria accounts for about two-thirds of outpatient visits in hospitals [36]. As mentioned earlier, more than $50 \%$ of out-patients wait for between one to three hours to see a doctor as a result of shortage of medical prescribers $[16,17,19]$. Therefore, authorizing pharmacists to clinically diagnose and prescribe for patients with uncomplicated malaria could provide prompt access to malaria treatment. However, given pharmacists inadequate skills in diagnosis, additional training would be needed. Hence, this study was followed-up with a survey conducted among Nigerian pharmacists to determine their areas of training needs in order to take on a prescribing role. 
Community pharmacists in Nigeria appear to be well positioned for prescribing considering their accessibility especially in urban areas, current counter prescribing practice and the reliance on them by members of the public for their treatment needs [25]. However, introducing prescribing in community pharmacies is subject to a number of challenges. These include lack of collaboration between community pharmacists and doctors and lack of access to patients' clinical records since there is no structure in place that would facilitate exchange of patients' clinical data between community pharmacies and hospitals. Therefore, a prescribing model likely to be successful in Nigerian community pharmacies is a limited form of independent prescribing using approved formularies or standard treatment guidelines for minor conditions. This was the type of prescribing model community pharmacists were authorised to do in South Africa in order to fill geographical gaps in relation to access to medical prescriber in rural settings [37]. Previous studies in Nigeria have advocated policy change to allow community pharmacists to treat minor disease conditions in Nigeria [25, 38]. Hence, utilising community pharmacists to provide primary healthcare in a similar manner to South Africa, offers an opportunity to increase patients access to care particularly in communities underserved by primary health centres. It also offers the opportunity to revitalise the current primary health care system in Nigeria which has been described as ineffective, and could serve as an incentive for community pharmacies presence in rural areas [37, 39]. Nevertheless, additional training would be needed to prepare community pharmacists to independently diagnose and treat disease conditions using standard treatment guidelines at the primary healthcare level.

Other barriers to pharmacist prescribing identified were organisational including shortage of pharmacists and pharmacists' lack of time to take on additional role. This is unsurprising considering that there are approximately 17,000 pharmacists in Nigeria which represents about 10 pharmacists to 100,000 people [40]. However, evidence from England suggests that utilising trained pharmacy technicians in some medicine supply roles of pharmacists, could free up pharmacists' time for extended clinical roles including prescribing [22]. This may represent a feasible option to address pharmacists' shortage and lack of time to do extended clinical roles in Nigeria.

This study has a number of limitations. First, the study employed telephone interviews which have certain limitations including their inability to observe non-verbal communication. However, the use of telephone interviews enabled the interviewing of participants across many locations in Nigeria. In addition, the quality and depth of data obtained was not compromised since the researcher was experienced in telephone interviews and data collection continued until saturation was reached. Secondly, being a qualitative study, the findings of this study cannot be statistically generalised. In addition, a small number of patient group representatives and general practitioners participated in the study. Therefore, we followed up this study with a 
quantitative survey to make our findings generalisable. Thirdly, one of the researchers in this study is a Nigerian pharmacist, who is to a large extent, experienced with pharmacy practice in Nigeria. This experience might have influenced key decisions made regarding this study including study aim and data interpretations. However, deliberate steps were taken including the triangulation of data sources and peer-reviewing of identified themes and data interpretations in order to ensure the 'trustworthiness' of the research. Finally, this study has provided avenues for future research including how best to: prepare pharmacists for a potential role in prescribing and promote collaboration between community pharmacists and doctors in Nigeria.

\section{Conclusion}

In conclusion, even though this study shows a difference in opinions among stakeholders regarding pharmacist prescribing, it clearly indicates that pharmacist prescribing would be well supported by many stakeholders including pharmacists and patients in Nigeria. In addition, our study reveals that pharmacist prescribing represents an opportunity to increase patients' access to treatment and the utilisation of pharmacists' skills in Nigeria. Nevertheless, a number of potential barriers that could hinder the introduction of pharmacist prescribing in Nigeria were identified including pharmacist inadequate skills in diagnosis, medical resistance and shortage of pharmacist.

\section{Acknowledgement}

The authors would like to thank all participants for their valuable time and contributions to this research.

\section{Funding}

No financial support was obtained for this study.

\section{Conflict of interest}

The authors do not have any conflict of interest to declare.

\section{References}

1. Review of prescribing, supply \& administration of medicines. Final report. 1999. http://webarchive.nationalarchives.gov.uk/20130107105354/http://www.dh.gov.uk/pro d_consum_dh/groups/dh_digitalassets/@dh/@en/documents/digitalasset/dh_407715 3.pdf. Accessed 30 March 2016. 
2. Kroezen M, Van Dijk L, Groenewegen PP, Francke AL. Nurse prescribing of medicines in Western European and Anglo-Saxon countries: a systematic review of the literature. BMC Health Serv Res. 2011;11:127.

3. Stewart D, MacLure K, George J. Educating nonmedical prescribers. $\mathrm{Br} \mathrm{J}$ Clin Pharmacol. 2012;74(4):662-667.

4. Bhanbhro S, Drennan VM, Grant R, Harris R. Assessing the contribution of prescribing in primary care by nurses and professionals allied to medicine: a systematic review of literature. BMC Health Serv Res. 2011;11:330.

5. Tonna, A.P., D. Stewart, and D. McCaig, An international overview of some pharmacist prescribing models. Journal of the Malta College of Pharmacy Practice, 2008(14):2026.

6. Pharmacy Council of New Zealand. Pharmacist Prescribers. http://www.pharmacycouncil.org.nz/cms display.php?st=1\&sn=232. Accessed 30 March 2016.

7. Yariv $\mathrm{H}$. The case of pharmacist prescribing policy in Israel. Israel Journal of Health Policy Research. 2015;4(1):49.

8. Nissen L. Hospital talk: Pharmacist prescribing-the perfect storm? Australian Journal of Pharmacy. 2014;95:29.

9. National Drug Policy-Nigeria. Abuja: Federal Ministry of Health, Nigeria and World Health Organisation; 2005.

10. Ehiri JE, Oyo-Ita AE, Anyanwu EC, Meremikwu MM, Ikpeme MB. Quality of child health services in primary health care facilities in south-east Nigeria. Child Care Health Dev. 2005;31(2):181-91.

11. Access to and rational use of medicines at the facility level. Abuja: Federal Ministry of Health, Nigeria; 2010.

12. Babalola CP, Awoleye SA, Akinyemi JO, Kotila OA. Evaluation of prescription pattern in Osun State (Southwest) Nigeria. Journal of Public Health and Epidemiology. 2011;3(3): 94-98.

13. Fagbule D, Kalu A. Case management by community health workers of children with acute respiratory infections: implications for national ARI control programme. J Trop Med Hyg. 1995;98(4):241-6.

14. Akande T. Referral system in Nigeria: Study of a tertiary health facility. Ann Afr Med. 2004;3(3):130-133.

15. Oyedeji R, Abimbola S. How tertiary hospitals can strengthen primary health care in Nigeria. Nig Med J. 2014;55(6):519-520.

16. Umar I, Oche MO, Umar AS Patient waiting time in a tertiary health institution in Northern Nigeria. Journal of Public Health and Epidemiology, 2011. 3(2):78-82. 
17. Ajayi IO. Patients' waiting time at an outpatient clinic in Nigeria--can it be put to better use? Patient Educ Couns. 2002;47(2):121-6.

18. World Bank. Physicians (per 1,000 people). http://data.worldbank.org/indicator/SH.MED.PHYS.ZS. Accessed 30 March 2016.

19. Oche M, Adamu H. Determinants of patient waiting time in the general outpatient department of a tertiary health institution in north Western Nigeria. Annals of Medical and Health Sciences Research. 2014; 3(4):588-592.

20. A lo A. Pharmacy in Nigeria. Am J Health Syst Pharm. 2006;63:670-673.

21. WAPCP Listing of fellows of the college (1991 - 2011). http://www.wapcp.info/sites/default/files/news attachments/wapcp listing of fellows 1991-2011.pdf. Accessed 1 February 2013

22. Auta A, Maz J, Strickland-Hodge B. Perceived facilitators to change in hospital pharmacy practice in England. Int J Clin Pharm. 2015;37(6):1068-75.

23. Erhun WO, Osigbesan T, Awogbemi JK. Study of pharmacists' and physicians' views on pharmacists prescribing in Nigeria. West African Journal of Pharmacy. 2013; 24(2):76-82.

24. Auta A, Bala ET, Shalkur D. Generic medicine substitution: a cross-sectional survey of the perception of pharmacists in North-Central, Nigeria. Med Princ Pract. 2014;23(1):53-8.

25. Auta, A., Fredrick NC, David S, Banwat SB, Adeniyi MA. Patients' views on their consultation experience in community pharmacies and the potential prescribing role for pharmacists in Nigeria. Journal of Pharmaceutical Health Services Research, 2014; 5:233-236.

26. Braun V, Clarke V. Using thematic analysis in psychology. Qual Res Psychol. 2006;3(2):77-101.

27. De Vries T, Henning RH, Hogerzeil HV, Fresle DA. Guide to Good Prescribing. A Practical Manual. Geneva: World Health Organization Action Programme on Essential Drugs, 1994.

28. Nissen L. Pharmacist prescribing: what are the next steps? Am J Health Syst Pharm. 2011; 68(24):2357-61.

29. Kondro W. Canada's doctors assail pharmacist prescribing. CMAJ. 2007; 177(6):558.

30. Gilbert L. Pharmacy's attempts to extend its roles: a case study in South Africa. Soc Sci Med. 1998;47(2):153-64.

31. Britten, N., Prescribing and the defence of clinical autonomy. Sociol Health Illn. 2001; 23(4):478-496.

32. Eaton, G. and B. Webb, Boundary encroachment: pharmacists in the clinical setting. Sociol Health IIIn. 1979; 1(1): 69-89. 
33. South African Pharmacy Council. Scope of practice and qualification for authorised pharmacist prescriber. Board Notice 122 of 2011. Government Gazette No. 34428.

34. Stewart DC, George J, Bond CM, Diack HL, McCaig DJ, Cunningham S. Views of pharmacist prescribers, doctors and patients on pharmacist prescribing implementation. Int J Pharm Pract. 2009;17(2):89-94.

35. Courtenay M, Carey N, Stenner K, Lawton S, Peters J. Patients' views of nurse prescribing: effects on care, concordance and medicine taking. $\mathrm{Br} \mathrm{J}$ Dermatol. 2011;164(2):396-401.

36. Nigeria Demographic and Health Survey 2008. Abuja: National Population Commission and ORC Macro, 2009.

37. Ward K, Sanders D, Leng H, Pollock AM. Assessing equity in the geographical distribution of community pharmacies in South Africa in preparation for a national health insurance scheme. Bull World Health Organ. 2014;92(7):482-9

38. Oparah AC, Arigbe-Osula EM. Evaluation of community pharmacists' involvement in primary health care. Trop J Pharm Res. 2002; 1(2):67-74.

39. World Health Organization. Nigeria still searching for right formula. 2015. http://www.who.int/bulletin/volumes/86/9/08-020908/en/. Accessed 22 June 2015.

40. Pharmacists Council of Nigeria. Full registration list of Pharmacists as at 2nd October, 2014. http://pcn.gov.ng/WEB\%20full\%20registration\%20list.pdf. Accessed 11 November 2014. 